\title{
Zwischen Empirie und Evidenz - (Re)Aktivierung der Veterinärphytotherapie
}

\author{
Michael Walkenhorst ${ }^{\mathrm{a}} \quad$ Christian R. Vogl ${ }^{\mathrm{b}} \quad$ Brigitte Vogl-Lukasser $^{\mathrm{b}} \quad$ Sabine Vollstedt ${ }^{\mathrm{c}}$ \\ Cäcilia Brendieck-Worm ${ }^{d}$ Silvia Ivemeyer ${ }^{e}$ Franziska Klarer $^{f}$ Beat Meier $^{f}$ \\ Kathrin Schmid ${ }^{g}$ Monika Disler ${ }^{g}$ Tinetta Bischoff ${ }^{g}$ Matthias Hamburger ${ }^{g}$ \\ Stephan Häsler ${ }^{\text {h }}$ Elisabeth Stöger ${ }^{i}$ \\ a Departement für Nutztierwissenschaften, Forschungsinstitut für biologischen Landbau (FiBL), Frick, Schweiz, \\ ${ }^{b}$ Institut für Ökologischen Landbau, Department für Nachhaltige Agrarsysteme, Universität für Bodenkultur, Wien, Österreich, \\ ${ }^{c}$ Bokholt-Hanredder, \\ ${ }^{\mathrm{d}}$ Niederkirchen, \\ ${ }^{e}$ Fachgebiet Nutztierethologie und Tierhaltung, Fachbereich Ökologische Agrarwissenschaften, Universität Kassel, Witzenhausen, \\ Deutschland, \\ ${ }^{\mathrm{f}}$ Institut für Biotechnologie, Departement Life Sciences und Facility Management, Zürcher Hochschule für Angewandte \\ Wissenschaften ZHAW, Wädenswil, \\ ${ }^{g}$ Department of Pharmaceutical Sciences, University of Basel, \\ ${ }^{\text {h }}$ Schweizerische Vereinigung für Geschichte der Veterinärmedizin, Gasel, Schweiz, \\ ' Feldkirchen, Österreich
}

Die Arbeit in der tierärztlichen Praxis findet tagtäglich im Spannungsfeld zwischen der eigenen Erfahrung und (neuesten) wissenschaftlichen Erkenntnissen statt. Im Vergleich zur Humanmedizin findet sich der Tiermediziner, unabhängig von der Therapierichtung, die er bevorzugt einsetzt, jedoch wesentlich öfter in der Situation, Entscheidungen ohne verfügbare wissenschaftliche Evidenz treffen zu müssen [1]. Die Empirie hat folglich nach wie vor einen besonderen Stellenwert im tierärztlichen Praxisalltag.

Aus dem Bedarf systematischer Dokumentation und Aufbereitung des traditionellen Erfahrungswissens hat sich ein eigenes Forschungsfeld entwickelt, das interdisziplinär sozial- und naturwissenschaftliche Methoden verbindet, und zu dem beispielsweise die Ethnobotanik, die Ethnopharmakologie oder auch die Ethnoveterinärmedizin zählen. Wo ist nun traditionelles Erfahrungswissen zur Veterinärphytotherapie zu erwarten? Einerseits in Asien, beispielsweise in China, wo die traditionell chinesische Veterinärmedizin seit Jahrtausenden ununterbrochen bis heute die Basis der dortigen tierärztlichen Tätigkeit ist. Und in Europa? Hier ist diese doch zumindest auf Jahrhunderten tierärztlicher Erfahrung beruhende Therapieform nahezu erloschen, doch noch gar nicht allzu lang, wie sich an Typoskripten veterinärpharmakologischer Vorlesungen aus der Mitte des vergangenen Jahrhunderts ablesen lässt [2]. Auch sonst ist das Erfahrungswissen der traditionell europäischen Veterinärmedizin nicht gänzlich verloren, ist es doch zumindest in Büchern erhalten geblieben [3]. Dennoch lässt das Studium dieser historischen Literatur immer wieder den Wunsch aufkeimen, noch einmal genau nachzufragen, wie einzelne Arzneipflanzen ganz konkret angewendet wurden, und es ist bedauerlich, dass die damaligen Therapeuten hierfür nicht mehr zur Verfügung stehen.

Und doch ist die Kräuteranwendung beim Tier in Europa gegenwärtig. Bäuerinnen und Bauern haben diese Tradition bis heute erhalten. Dank ihrer großzügigen Kooperationsbereitschaft konnten in den vergangenen 10 Jahren in den beiden Alpenländern Österreich und Schweiz ethnoveterinärmedizinische Forschungsprojekte erfolgreich abgeschlossen werden [4-6], und weitere laufen aktuell oder sind in Planung.

Es besteht also eine solide Basis, um die Veterinärphytotherapie zu aktivieren und somit das reichhaltige Potential der Arzneipflanzen zukünftig in der veterinärmedizinischen Forschung, Lehre und Praxis wieder verstärkt zu nutzen. Mit dem Parallelsymposium Veterinärmedizin möchten die Organisatoren der Tagung «Phytotherapie 2014» genau dies aktiv anregen.

Michael Walkenhorst

\section{KARGER}

Fax +497614520714

Information@Karger.com

www.karger.com
(C) 2014 S. Karger GmbH, Freiburg 


\section{Literatur}

1 Cockcroft PD, Holmes MA (eds): Handbook of Evidence-based Veterinary Medicine. Oxford, Blackwell Publishing, 2003, pp 2-7.

2 Steck W: Pharmakologie. Typoskript, erstellt von Gross R, Bern, 1944. (Kopie im Archiv der Schweizerischen Vereinigung für Geschichte der Veterinärmedizin).

3 von Grebner J, von Straub GA: Thierärztliches Recept-Taschenbuch, ed 4. Ulm, J. Ebner'sche Buchhandlung, 1883
4 Vogl-Lukasser B, Vogl CR, Bizaj M, Grasser S, Bertsch C: Lokales Erfahrungswissen über Pflanzenarten aus Wildsammlung mit Verwendung in der Fütterung und als Hausmittel in der Volksheilkunde bei landwirtschaftlichen Nutztieren in Osttirol. Endbericht zum ProjektNr. 1272, GZ 21.210/41-II1/03 (Teil 1), gefördert vom Land Tirol und dem Lebensministerium (BM:LFUW). 2006.

5 Schmid K, Ivemeyer S, Vogl CR, Klarer F, Meier B, Hamburger M, Walkenhorst M: Traditional use of herbal remedies in livestock by farmers in 3 Swiss cantons (Aargau, Zurich, Schaffhausen). Forsch Komplementmed 2012; 19:125-136.

6 Disler M, Ivemeyer S, Hamburger M, Vogl CR Tesic A, Klarer F, Meier B, Walkenhorst M: Ethnoveterinary herbal remedies used by farmers in four north-eastern Swiss cantons (St. Gallen, Thurgau, Appenzell Innerrhoden and Appenzell Ausserrhoden). Ethnobiol Ethnomed 2014;10:32.

\section{Erfahrung schafft Wissen - Einführung in die ethnoveterinärmedizinische Forschung}

\author{
Autoren: Christian R. Vogl, Brigitte Vogl-Lukasser, \\ Michael Walkenhorst
}

\section{Einleitung}

Weltweit halten Millionen von Bäuerinnen und Bauern Tiere, ohne auf moderne chemisch-synthetische Tierarzneimittel oder Tierärzte zugreifen zu können. In der Vorbeugung und Behandlung ihrer Tiere setzen sie seit Jahrhunderten Hausmittel - vielfach pflanzlichen Ursprungs - ein und entwickeln diese empirisch weiter. Die auf diese Weise entstandenen Hausmittel beruhen somit auf Erfahrungen, die während zahlreicher Generationen erworben wurden. Die Ethnoveterinärmedizin (EVM) ist die Forschungsrichtung, die unter anderem diese Hausmittel und die dazu gesammelten Erfahrungen dokumentiert und untersucht $[1,2]$.

\section{Methoden}

Wissenschaftler, die zu Themen der EVM arbeiten, nutzen eine breite Palette an Methoden, wie sie auch z.B. für die Ethnobotanik [3-5] oder Medical Anthropology, Ethnomedizin sowie Ethnopharmakologie [6] angewendet werden. Im Falle der EVM werden Wissen, Verhalten, Wahrnehmungen sowie Werte und Einstellungen von Gesprächspartner zu Management, Wohlbefinden, Krankheit und Behandlung von Tieren durch unterschiedliche Arten von sozialwissenschaftlichen Erhebungsmethoden (z.B. Fragebögen, teilstrukturierte Interviews, teilnehmende Beobachtung, Cultural Domain Analysis inklusive free lists, pile sorts, rankings, ratings, pairwise comparison) dokumentiert und mit naturwissenschaftlichen Elementen kombiniert. Im Vordergrund stehen in bisher durchgeführten Arbeiten nicht die naturwissenschaftlichen Methoden zur Beweisführung über eine allfällige Wirkung, sondern das sorgfältige und detaillierte Erfassen des «Erfahrungswissens» der Gesprächspartner. Erfahrungswissen wird dabei nach Berkes [traditional ecological knowledge, 7] als ein Komplex aus kog- nitivem Wissen, Handfertigkeiten (frei übersetzt für implicit knowledge, practice), sozialer Organisation des Handelns, Kosmovision sowie mit dem Handeln verknüpfter sprachlicher Ausdrücke verstanden. Die Erhebungsinstrumente werden darauf abgestimmt, welche dieser genannten Aspekte untersucht werden sollen.

\section{Anwendung ethnoveterinärmedizinischer Forschungsme- thoden in Mitteleuropa}

EVM-Forschungsprojekte in Österreich und in der Schweiz [z.B. 8, 9] zeigen, dass der Kanon an verfügbaren Methoden sehr gut in der Lage ist, auch in Mitteleuropa EVM-Erfahrungswissen zu dokumentieren. Bisher wurden diese Arbeiten als wissenschaftliche Abschlussarbeiten agrarischer oder pharmazeutischer Studiengänge sowie als kompetitive Forschungsprojekte durchgeführt. Hierbei gilt es eine Reihe von Herausforderungen zu meistern:

Im Vergleich zu kulturanthropologischen Studiengängen werden in naturwissenschaftlichen Ausbildungen kaum sozialwissenschaftliche Erhebungsmethoden gelehrt. Es bedarf daher eines hohen Aufwands, die Mitarbeiter methodisch im Einsatz präziser sozialwissenschaftlicher Erhebungsinstrumente zu schulen. Insbesondere müssen suggestive Fragestellungen (not imposing concepts) vermieden und klar zwischen Wissen auf der einen und Verhalten auf der anderen Seite differenziert werden.

Als Herausforderung wurde auch wahrgenommen, dass immer wieder einzelne von den Gesprächspartnern beschriebene Hausmittelanwendungen - wissenschaftlich begründet oder aber auch wissenschaftlich unbegründet - nicht im Einklang mit den gesetzlichen Rahmenbedingungen stehen. Hier gilt es, eine stabile Vertrauensbeziehung zu etablieren, um diese Informationen dennoch zu erhalten. Zudem muss zwingend die Anonymität gewahrt bleiben. Insbesondere bei wissenschaftlich begründeten Bedenken im Hinblick auf die Anwendung einzelner Hausmittel entsteht ein Spannungsverhältnis zwischen dem Wunsch nach Darlegung der erhaltenen Ergebnisse und der Sorge um Risiken für potentielle Nachahmer. Solche Ergebnisse dürfen selbstverständlich nicht ohne eine entsprechende Kommentierung publiziert werden. 


\section{Schlussfolgerung}

Vor dem Hintergrund der bereits bestehenden (z.B. in der Biologischen Landwirtschaft) sowie der zu erwartenden zunehmenden Restriktionen für den Einsatz schulmedizinischer Tierarzneimittel (z.B. Antibiotika) in der Tierhaltung sollte die Ethnoveterinärmedizin eine wesentliche Rolle bei der Weiterentwicklung präventiver und therapeutischer Konzepte für Tiere spielen. Der sehr große Erfahrungsschatz von Bäuerinnen und Bauern im Einsatz von Hausmitteln über die vergangenen Jahrhunderte schafft genau so Wissen, wie die Praxis erfahrener Tierärztinnen und Tierärzte. Bäuerinnen und Bauern müssen stärker als bisher als kompetentes Gegenüber in der Planung und Umsetzung präventiver und therapeutischer Maßnahmen wahrgenommen werden. Zu Wünschen wäre, dass Erfolg versprechende Anwendungen von Hausmitteln in Folgeprojekten von der veterinärmedizinischen Forschung getestet werden, in der Ausbildung von Tierärztinnen und Tierärzten das Thema «Hausmittel» verstärkt aufgegriffen wird und sich Beratungsprojekte speziell diesem Thema widmen.

\section{Literatur}

1 Martin M, Mathias E, McCorkle C (eds): Ethnoveterinary Medicine: An Annotated Bibliography of Community Animal Healthcare. London, ITDG Publishing, 2001.

2 McCorkle CM, Mathias E, Schillhorn van Veen TW (eds): Ethnoveterinary Research and Development. London, Intermediate Technology Publications, 1996.

3 Martin G: Ethnobotany - a Methods Manual. London, Earthscan, 2004

4 Cotton VM: Ethnobotany - Principles and Applications. Chichester, John Wiley \& Sons, 1996.

5 Alexiades MN, Sheldon JW (eds): Selected Guidelines for Ethnobotanical Research: A Field Manual. New York, New York Botanical Garden, 1996.

6 Berlin EA, Berlin B: Some field methods in medical ethnobiology. Field Methods 2005;17:235-268.

7 Berkes F: Sacred Ecology - Traditional Ecological Knowledge and Resource Management. Philadelphia, Taylor and Francis, 1999.

8 Vogl-Lukasser B, Vogl CR, Bizaj M, Grasser S, Bertsch C: Lokales Erfahrungswissen über Pflanzenarten aus Wildsammlung mit Verwendung in der Fütterung und als Hausmittel in der Volksheilkunde bei landwirtschaftlichen Nutztieren in Osttirol. Endbericht zum ProjektNr. 1272, GZ 21.210/41-II1/03 (Teil 1), gefördert vom Land Tirol und dem Lebensministerium (BM:LFUW), 2006

9 Schmid K, Ivemeyer S, Vogl CR, Klarer F, Meier B, Hamburger M, Walkenhorst M: Traditional use of herbal remedies in livestock by farmers in 3 Swiss cantons (Aargau, Zurich, Schaffhausen). Forsch Komplementmed 2012;19:125-136.

\section{Ursprung und Aktualität der \\ Kräuteranwendungen in der Traditionellen Chinesischen Veterinärmedizin}

\author{
Autor: Sabine Vollstedt
}

\section{Einleitung}

Die chinesischen Kräuter in der Traditionell Chinesischen Veterinärmedizin werden seit etwa 4000 Jahren eingesetzt. Es fanden sich Inschriften auf Knochen schon in der Shang Dynastie (16. bis 11. Jh.v.Chr.), in denen Erkrankungen bei Menschen und Tieren beschrieben wurden. Seit der Zeit der streitenden Reiche (475-221 v.Chr.) gab es spezialisierte Tierärzte, und aus der Zeit der Han Dynastie (206-220 n.Chr.) fanden sich tierärztliche Rezepte auf Bambus. Diese Tradition der Schriftführung wurde über die Jahrhunderte beibehalten, und somit haben wir heute ein umfangreiches Wissen über die traditionelle Anwendung chinesischer Pflanzen [1,2].

\section{Charakterisierung der chinesischen Kräuter mit Bezug zu wissenschaftlichen Erkenntnissen}

Die Charakterisierung der Pflanzen in Bezug auf Geschmack, Temperaturverhalten und Organspezifität spielen für die Behandlung eine große Rolle. Die Diagnostik erfasst Kriterien, die eine ganzheitliche Einschätzung der Erkrankung ermöglichen. Das Behandlungskonzept basiert auf dieser Diagnose. Inzwischen gibt es wissenschaftliche Belege, dass diese Kriterien auch heute noch ihre Bedeutung haben [3, 4].

Astragalus membranaceus Schischkin (Huang Qi) ist ein wichtiger Bestandteil vieler Rezepturen, die in der Veterinärmedizin eingesetzt werden. Es ist ein Qi-Tonikum und gilt als warm mit süßem Geschmack. Ein Einsatzgebiet ist die Behandlung von Defizienzen des Immunsystems, dem Wei Qi. Die heutige Forschung kann genau aufzeigen, welche Effekte, A. membranaceus Schischkin auf das Immunsystem hat. Die Beeinflussung ist vielseitig und mehrschichtig. Es ist nicht nur ein Aktivator von Immunzellen, sondern kann bei überschieBenden Reaktionen auch regulativ wirksam werden [5-7]. Somit beeinflusst $A$. membranaceus Schischkin das Immunsystem modulierend und sorgt so für eine optimale Immunantwort.

Angelica sinensis (Oliv.) Diels (Dang Gui) ist ein Blut-Tonikum mit warmem Temperaturverhalten und süßem, leicht scharfem Geschmack. Es wurde über Jahrtausende bei postpartalen Störungen der Frau eingesetzt. Wissenschaftliche Ergebnisse zeigen, dass $A$. sinensis in Mausmodellen des Blutmangels einen positiven Einfluss hat [8]. Es hat eine immunmodulierende Wirkung und sorgt für eine ausreichende Bereitstellung von Zellen, auch bei Infektionen mit hohem Bedarf an aktivierten Zellen $[9,10]$. In der heutigen Zeit wird es 
bei unspezifischen Symptomen wie allgemeiner Müdigkeit und Leistungsverminderung mit blassen Schleimhäuten eingesetzt. Dieser Symptomenkomplex wird in der TCM als MilzQi, bzw. Blutmangel beschrieben und beinhaltet häufig auch eine herabgesetzte Funktion des Immunsystems.

Es ist faszinierend, wie die Chinesen in einer Zeit ohne Mikroskop, ohne chemische und biologische Tests es schafften, relativ genaue Beobachtungen so zu formulieren, dass man sie mit den heutigen wissenschaftlichen Methoden bestätigen kann. Diese Synergie von Empirie und Wissenschaft ist hoch aktuell und für beide Seiten von Nutzen. Durch mehr Detailwissen aus pharmazeutischer und klinischer Forschung können Behandlungen einerseits optimiert und andererseits erfolgreiche traditionelle Behandlungsstrategien genutzt werden, um auf wissenschaftlichem Niveau gezielt nach den Wirkungsmechanismen zu suchen. Traditionelles Wissen und moderne Wissenschaften zusammenzubringen ist eine Kunst, die wahres Wissen hervorbringt.

\section{Literatur}

1 Bensky D, Clavey S, Stöger E: Chinese Herbal Materia Medica. Vista, Eastland Press, 2004.

2 Xie HS, Preast V: Xie's Chinese Veterinary Herbology. Hoboken, Wiley Blackwell, 2010.

3 Zhao YL, Wang JB, Xiao XH, Zhao HP, Zhou CP: Study on the cold and hot properties of medicinal herbs by thermotropism in mice behavior. J Ethnopharmacol 2011;16:980-985.

4 Liao H, Branbury LK, Leach DN: Antioxidant activity of 45 Chinese herbs and the relationship with their TCM characteristics. Evid Based Complement Alternat Med 2008;5:429-434.

5 Zhao LH, Ma ZX, Zhu J, Yu XH, Weng DP: Characterization of polysaccharide from Astragalus radix as the macrophage stimulator. Cell Immunol 2011;271:329334.

6 Chen R, Shao H, Lin S, Zhang JJ, Xu KQ: Treatment with Astragalus membranaceus produces antioxidative effects and attenuates intestinal mucosa injury induced by intestinal ischemia-reperfusion in rats. Am J Chin Med 2011;39:879-887.

7 Huang GC, Wu LS, Chen LG, Yang LL, Wang CC: Immuno-enhancement effects of Huang Qi Liu Yi Tang in a murine model of cyclophosphamide-induced leucopenia. J Ehnopharmacol 2007;19:229-235.

8 Li W, Tang Y, Guo J, Huang M, Li W, Qian D, Duan J: Enriching blood effect comparison in three kinds of blood deficiency model after oral administration of drug pair of Angelica Sinensis Radix and Chuanxiong Rhizoma and each single herb (article in Chinese). Zhongguo Zhong Yao Za Zhi 2011;36:1808-1814.

9 Chen Y, Duan JA, Qian D, Gui J, Song B, Yang M: Assessment and comparison of immunoregulatory activity of four hydrosoluble fractions of Angelica sinensis in vitro on the peritoneal macrophages in ICR mice. Int Immunopharmacol 2010;10:422-430.

10 Queiroz ML, Torello CO, Constantino AR, Ramos AL, Queiroz J: Angelica sinensis modulates immunohematopoietic response and increases survival of mice infected with Listeria monocytogenes. J Med Food 2010;13:1451-1459.

\section{Auf der Strecke geblieben? Auf den Spuren der Kräuteranwendungen in der traditionellen europäischen Veterinärmedizin}

\author{
Autorin: Cäcilia Brendieck-Worm
}

\section{Einleitung}

Die Recherche in antiquarischer veterinärmedizinischer Literatur zeigt, dass Heilpflanzen in der europäischen Tierheilkunde eine lange Tradition haben und viele seit Jahrhunderten bei gleicher Indikation angewendet wurden. In der aktuellen schulmedizinischen Fachliteratur fehlen sie jedoch weitgehend oder werden als obsolet bezeichnet, dies auch dort, wo die Therapie mit synthetischen Medikamenten unbefriedigend ist, so z.B. bei funktionellen Magen-Darmstörungen.

\section{Traditionelle europäische Veterinärmedizin funktioneller Magen-Darmstörungen}

Noch vor 100 Jahren waren Ätherischöldrogen wie Kümmel und Bitterstoffdrogen wie Enzian zur Therapie von Verdauungsstörungen selbstverständlich.

Aus der sogenannten Stallmeisterzeit (1250-1750 n.Chr.) sind etliche Enzian enthaltende Rezepte überliefert, so in Nachrichters Roß-Arzneybuch: Der Scharfrichter Johannes Deigendesch empfiehlt Enzian zur Stärkung des Magens, bei Wurmbefall und in Rezepturen, die «sonderheitlich zu Erhaltung der Gesundheit» dienen. Enzian fehlt auch nicht im «Ordinari Viehpulver» und im «Viehtheriac», die nicht nur zur «Curirung», sondern auch präventiv eingesetzt wurden [1].

Im «Thierärztlichen Recept-Taschenbuch» des Militärobertierarztes v. Grebner und Obermedizinalrates Professor von Straub (1883) für Tierbesitzer und Tierärzte wird Enzian in Rezepturen gegen Überfütterungs- und Entzündungskoliken und chronischen Magen-Darmkatarrh bei Pferd und Wiederkäuer empfohlen [2].

Auch im 1898 erstmals erschienenen «Lehrbuch der Arzneimittellehre für Thierärzte» von Prof. Fröhner [3] wird Enzian in Rezepturen gegen Verdauungsschwäche, Indigestion und Magendarmkatarrh erwähnt, ebenso in «Dr. Lampe's illustrierte Tierheilkunde» (1899), einem «ärztlichen Hausschatz für sämtliche Krankheiten» [4].

Noch in der für Apotheker konzipierten Sammlung «Tierarznei-Rezepte» von Döderlein (1968) sind Enzian-haltige Kolik- und Wiederkäumixturen, Rezepte gegen Trommelsucht der Kaninchen und appetitanregende «Mastpulver» für unter anderem Schweine aufgeführt [5].

Auch der Kümmel ist in diesen Werken in Rezepturen bei Magen-Darmstörungen oder zur Stärkung der Verdauung ver- 
treten. Fröhner bezeichnet ihn als Hauptrepräsentant der «Carminativa» und bescheinigt ihm blähungstreibende, krampfstillende und gärungswidrige Wirkung, was ihn zur Anwendung bei Kolik, Tympanie und abnormer Gärung prädestiniert [3].

\section{Einsatz in der modernen Tierarztpraxis}

Während die traditionellen Heilpflanzen als zugelassene Medikamente dem Tierarzt praktisch nicht mehr zur Verfügung stehen, erleben sie eine Renaissance als Ergänzungsfuttermittel. Diese sind jedoch in der Mehrzahl zur Anwendung durch den Laien konzipiert.

Inwieweit Heilpflanzen als Veterinärtherapeutika ernst genommen werden, ist wesentlich vom Selbstverständnis des Tierarztes abhängig.

Zwar fehlen in der Tiermedizin weitgehend moderne klinische Studien, in denen die empirisch entwickelten Heilpflanzenrezepturen wissenschaftlich geprüft worden wären. Aus Sicht des Praktikers spricht jedoch kaum etwas gegen den Einsatz empirisch als wirksam befundener Pflanzenzubereitungen. Aktuelle phytopharmazeutische Forschung und klinische Studien in der Humanmedizin bestätigen vielfach ihre Wirksamkeit. Kümmel, Enzian und viele weitere traditionell eingesetzte Heilpflanzen sind ESCOP-monografiert und damit wissenschaftlich anerkannt.

Warum also das Überlieferte zurückweisen, bietet es doch eine wichtige Therapieoption insbesondere im Übergangsbereich zwischen Prävention und Therapie, für den schulmedizinische Therapeutika weitgehend fehlen. Dieser Bereich hat gerade in der Tiermedizin an Bedeutung gewonnen. Und dies auch, weil Tiere durch zunehmende Entfremdung von ihrer natürlichen pflanzlichen Nahrungsgrundlage krankheitsanfälliger werden.

Heilpflanzen grundsätzlich dem Laienbehandler zu überlassen würde für die Tiermedizin einen erheblichen Verlust bedeuten. Der phytotherapeutisch geschulte Tierarzt mit seinen professionellen diagnostischen und therapeutischen Fähigkeiten ist prädestiniert zur Nutzen-Risiko-Abschätzung beim Heilpflanzeneinsatz und kann, wo es nötig sein sollte, synthetische und pflanzliche Präparate angemessen kombinieren. Hierfür qualitativ hochwertige, möglichst als Arzneimittel zugelassene Veterinärphytotherapeutika an die Hand zu bekommen, erwartet der Praktiker von Wissenschaft, Industrie und Politik.

\section{Literatur}

1 Deigendesch J: Nachrichters Roß-Arzneybuch. Frankfurt, 1785.

2 von Grebner J, von Straub GA: Thierärztliches Recept-Taschenbuch, ed 4. Ulm, J. Ebner'sche Buchhandlung, 1883.

3 Fröhner E: Lehrbuch der Arzneimittellehre für Thierärzte, ed 4. Stuttgart, Enke, 1896.

4 Schwarz A, Seyffert A: Dr. Lampe's illustrierte Tierheilkunde. Leipzig, Ernst Wiest Nachf., 1899.
5. Döderlein G (Stather-Döderlein): Tierarznei-Rezepte, ed 3. Stuttgart, Wissenschaftliche Verlagsgesellschaft, 1968.

\section{Ethnoveterinärmedizinische Forschung in der Schweiz}

\section{Autoren: Michael Walkenhorst, Silvia Ivemeyer, Franziska \\ Klarer, Beat Meier, Christian R. Vogl, Kathrin Schmid, \\ Monika Disler, Tinetta Bischoff, Matthias Hamburger}

\section{Einleitung}

Das traditionelle bäuerliche Erfahrungswissen zum Einsatz von Arzneipflanzen beim Nutztier birgt ein wichtiges Potential, zukunftsweisende Strategien für die Nutztiermedizin zu entwickeln. Abgesehen von Österreich fehlen ethnoveterinärmedizinische Studien für Mitteleuropa. Ein gemeinsam vom Forschungsinstitut für biologischen Landbau (FiBL, Frick), der Universität Basel und der Zürcher Hochschule für Angewandte Wissenschaften (ZHAW, Wädenswil) initiiertes Projekt hat sich 2011 zum Ziel gesetzt, das traditionelle bäuerliche Erfahrungswissen zum Einsatz von Arzneipflanzen beim Nutztier schweizweit mit einer einheitlichen Methodik zu erfassen. Fast unbemerkt begann die ethnoveterinärmdizinische Forschung in der Schweiz jedoch bereits schon einige Jahre zuvor in Form einer agronomischen Masterarbeit, die sich dem Safiental, einer Region in Graubünden, widmete. Insgesamt 30 Interviews wurden hier durchgeführt und bereits 84 Pflanzenarten dokumentiert [1]. Der gesamte Kanton Graubünden wurde dann 2011 in einer unter der Federführung der ZHAW durchgeführten Studie näher betrachtet. Im Folgenden sollen die 3 in den Jahren 2011-2013 durchgeführten pharmazeutischen Masterarbeiten [2-4] zusammenfassend dargestellt werden. Im Fokus standen 13 Kantone der Nord-, Nordost- und Zentralschweiz (Tab. 1).

\section{Methoden}

Verschiedene Vorgehensweisen kamen jeweils parallel zum Einsatz, um die Interviewpartnerinnen und -partner (IVP) für die Projekte zu finden. Alle IVP wurden persönlich besucht, um ihr Erfahrungswissen zu dokumentieren. Für jede genannte Rezeptur wurden detaillierte Informationen $\mathrm{zu}$ den verwendeten Pflanzen und zum Herstellungsprozess der anwendungsfertigen Präparate erfasst. Darüber hinaus wurden für deren Anwendungsgebiete Dosierung, Verabreichungsart, -frequenz und -dauer, Herkunft des Wissens, Einsatzhäufigkeit und die Zufriedenheit mit dem Ergebnis der Therapie dokumentiert. 
Tab. 1. Traditionelle pflanzliche Hausmittelrezepte der Nord-, Nordost- und Zentralschweiz und deren Anwendungen

\begin{tabular}{|c|c|c|c|c|c|c|}
\hline Jahr & Kantone & $\begin{array}{l}\text { Anzahl } \\
\text { Interviews } \\
\text { (Personen) }\end{array}$ & $\begin{array}{l}\text { Rezepte } \\
\text { mit je nur } \\
\text { einer } \\
\text { Pflanzenart }\end{array}$ & $\begin{array}{l}\text { Pflanzen- } \\
\text { arten }\end{array}$ & 4 häufigste Arten in absteigender Reihenfolge & Anwendungen \\
\hline 2011 & $\mathrm{AG}, \mathrm{ZH}, \mathrm{SH}$ & $21(24)$ & 123 & 43 & $\begin{array}{l}\text { Matricaria recutita L., Calendula officinalis L., } \\
\text { Symphytum officinale L., Coffea arabica L. }\end{array}$ & 150 \\
\hline 2012 & TG, SG, AI, AR & $38(50)$ & 315 & 76 & $\begin{array}{l}\text { Matricaria recutita L., Calendula officinalis L., } \\
\text { Rumex obtusifolius L., Urtica dioica L. }\end{array}$ & 428 \\
\hline 2013 & $\begin{array}{l}\text { GL, NW, OW, SZ, } \\
\text { UR, ZG }\end{array}$ & $49(63)$ & 230 & 68 & $\begin{array}{l}\text { Matricaria recutita L., Calendula officinalis L. } \\
\text { Utrica dioica } \mathrm{L} ., \text { Coffea arabica } \mathrm{L} .\end{array}$ & 278 \\
\hline ges. & 13 & 108 (137) & 668 & 109 & & 856 \\
\hline
\end{tabular}

\section{Resultate}

Es wurden 108 Interviews mit insgesamt 137 Personen geführt und 1025 Hausmittelrezepturen erfasst. Davon enthielten 668 Rezepturen je nur eine einzelne Pflanzenart (Tab. 1). Ausschließlich diese Rezepturen wurden in die Auswertung einbezogen. Die beiden meistgenannten der insgesamt 109 dokumentierten Pflanzenarten waren Matricaria recutita L. und Calendula officinalis L. Regional unterschiedlich belegten Coffea arabica L., Rumex obtusifolius L., Symphytum officinale L. und Urtica dioica L. den 3. und 4. Rang. Für jede Rezeptur wurden bis zu 4 verschiedene Anwendungen, überwiegend für Rinder, beschrieben. Die meistgenannten Anwendungsgebiete waren a) Hautveränderungen und Wunden sowie b) Erkrankungen des Magen-Darm-Traktes und Stoffwechselstörungen. Die beschriebenen Anwendungen wurden überwiegend topisch oder oral verabreicht. Mehr als die Hälfte der beschriebenen Anwendungen kamen innerhalb des letzten Jahres vor dem Interview zum Einsatz. Für insgesamt 18 Pflanzenarten konnten orale Tagesdosen, für 11 Pflanzenarten Konzentrationen in extern verabreichten Rezepturen auf der Basis von mindestens 3 unabhängig voneinander beschriebenen Anwendungen ermittelt werden.

\section{Schlussfolgerung}

Das lokale Erfahrungswissen über pflanzliche Hausmittel und deren Anwendungen bei Nutztieren ist in weiten Teilen der Schweiz vorhanden und basiert auf einer breiten Palette von Pflanzenarten. Die auf diesem Erfahrungswissen basierenden Rezepturen werden nach wie vor angewendet. Vor dem Hintergrund aktueller phytopharmazeutischer und klinisch-humanmedizinischer Forschungsergebnisse erscheint ein wesentlicher Teil der eingesetzten Rezepturen sinnvoll.

\section{Literatur}

1 Joos B: Lokales Wissen über Gesundheit und Krankheit des Viehs in Graubünden. Wien, Universität für Bodenkultur, 2010.
2 Schmid K, Ivemeyer S, Vogl CR, Klarer F, Meier B, Hamburger M, Walkenhorst M: Traditional use of herbal remedies in livestock by farmers in 3 Swiss cantons (Aargau, Zurich, Schaffhausen). Forsch Komplementmed 2012;9:25-136.

3 Disler M, Ivemeyer S, Hamburger M, Vogl CR, Tesic A, Klarer F, Meier B, Walkenhorst M: Ethnoveterinary herbal remedies used by farmers in four northeastern Swiss cantons (St. Gallen, Thurgau, Appenzell Innerrhoden and Appenzell Ausserrhoden). J Ethnobiol Ethnomed 2014;10:32.

4 Bischoff T: Plant and natural product based household remedies manufactured and used by farmers of six central Swiss cantons to treat livestock. Basel, University of Basel, 2013.

\section{Bewahrung des Wissens - eine Bestandsaufnahme im Kanton Graubünden}

\author{
Autorin: Franziska Klarer
}

\section{Einleitung}

Obwohl Wissen über die Anwendung von Hausmitteln im Kanton Graubünden noch vorhanden ist [1], drohen die traditionellen Kenntnisse zum Einsatz von Arzneipflanzen und Hausmitteln bei Tieren verloren zu gehen. Ziel der Studie war, im Kanton Graubünden noch vorhandenes, mündlich überliefertes Wissen zum Einsatz von Arzneipflanzen bei Rindern, Schafen, Ziegen, Schweinen und Pferden zu dokumentieren und Tierhaltern wieder zur Verfügung zu stellen.

\section{Methode}

Über das Landwirtschaftliche Bildungs- und Beratungszentrum Plantahof, Landquart, über praktizierende Bündner Großtierärzte sowie via Weiterempfehlungen wurden Gewährsleute rekrutiert. 35 wurden von März bis November 2011 interviewt, mit standardisierten Fragebögen zur Sammlung von Arzneipflanzen, zur Zubereitung und Anwendung von Hausmitteln (3 Interviews abgebrochen). Das Ziel einer praxisbezogenen Publikation für Tierhalter erforderte eine Beurteilung der Hausmittel, um die Weiterverbreitung von Fehlinformationen zu vermeiden. Anhand persönlicher Erfah- 
rung von 2 Gutachtern (Tierärztin/Pharmazeut) sowie Angaben aus der Fachliteratur wurden die pharmazeutische Zubereitung und die Verabreichung der Hausmittel, welche Teile von Pflanzen oder Flechten enthielten und von den Studienteilnehmern selbst angewendet wurden, in 4 Kategorien eingeteilt: empfohlen, bedingt empfohlen (benötigen eine Korrektur in Zubereitung oder Verabreichung), nicht beurteilbar, nicht empfohlen.

\section{Resultate}

407 Hausmittel wurden erfasst. Eine klare Abgrenzung zwischen mündlich überliefertem und an Heilpflanzenkursen erworbenem Wissen war nicht immer möglich. 287 Hausmittel und deren Anwendungen - insgesamt 346 (379 vor Zusammenzug einzelner Anwendungen) - wurden begutachtet. 225 Anwendungen konnten empfohlen, 53 bedingt empfohlen, 17 nicht beurteilt und 51 nicht empfohlen werden. Die beurteilten Hausmittel enthielten 122 verschiedene Ausgangsstoffe. $271 \mathrm{Mal}$ wurden Arzneidrogen genannt, $116 \mathrm{Mal}$ andere Bestandteile wie Alkohol hochprozentig (15), Rotwein (6), Essig (6), Ö1/Fett (14), Zucker (19), Salz (8), Nahrungs- und Futtermittel (20), Tabak (3), Asche/Seife (3), pharmazeutisch hergestellte Zubereitungen aus Arzneipflanzen (10) sowie Umgebungsbehandlungen (12) wie z.B. das Aufhängen von Zweigen und die Nutzung von Pflanzen für Stalleinrichtungen oder als Einstreu.

Tabelle 1 zeigt einen Vergleich der Hausmittelanwendungen (HM) mit der Anzahl Tierarzneimittel (TAM), die in der Schweiz zwischen 1924 und 2011 eine Zulassung hatten und pflanzliche Bestandteile enthielten [2]. Bei beiden Zählungen überwiegen die Anwendungen für den Gastrointestinaltrakt und Metabolismus. Bei den topischen Anwendungen weisen die Hausmittel auf einen hohen Bedarf hin. Dieser war auch früher nicht durch zugelassene Tierarzneimittel abgedeckt.

\section{Schlussfolgerung}

Traditionelle Hausmittel sollen nicht ohne weitere Abklärungen weiterempfohlen werden [3], da nur eine korrekte $\mathrm{Zu}$ bereitung und gezielte Verabreichung der Hausmittel Erfolg versprechend ist. Hausmittel für Haut, Klauen und Hufe, Euter und Zitzen sowie Muskel- und Skelettsystem, welche meist äußerlich angewendet werden, sind verbreitet. Diese einfach zu applizierenden Hausmittel müssen häufig wiederholt eingesetzt werden. Somit bringen sie den Vorteil, dass der Tierhalter den Heilungsverlauf verfolgen muss. Die Daten der Studie zeigen zudem das Potenzial von pflanzlichen Hausmitteln für Erkrankungen des Gastrointestinaltraktes und des Metabolismus sowie des Respirationstrakts. Generell liegt ein weites Forschungsfeld brach zur Reaktivierung der Phytotherapie in der Veterinärmedizin.

\section{Literatur}

1 Joos B: Lokales Wissen über Gesundheit und Krankheit des Viehs in Graubünden. Wien, Universität für Bodenkultur, 2010

2 Klarer F, Häsler S, Marusic-Bubenhofer R, Meier B: Zulassungen pflanzlicher Tierarzneimittel in der Schweiz 1924-2011. Z Phytother 2012;33(suppl 1):19-20. Datenbank einsehbar unter: www.ibt.zhaw.ch/phytopharmazie (F\&E/Dienstleistungen->Projekte->Projektliste->Pflanzliche Arzneimittel in der Tiermedizin).

3 Klarer F, Stöger E, Meier B: Jenzerwurz und Chäslichrut. Pflanzliche Hausmittel für Rinder, Schafe, Ziegen, Schweine und Pferde. Zürich, Haupt, 2013.

Tab. 1. Bündner Hausmittelanwendungen (HM) verglichen mit Zulassungen pflanzlicher Tierarzneimittel (TAM) in der Schweiz 1924-2011 pro Anwendungsgebiet sowie am häufigsten genannte Arzneidrogen in den als «empfohlen» und «bedingt empfohlen» begutachteten Hausmitteln

\begin{tabular}{|c|c|c|c|}
\hline Anwendungsgebiet & $\begin{array}{l}\text { Anzahl } \\
\text { HM }\end{array}$ & $\begin{array}{l}\text { Anzahl } \\
\text { TAM }\end{array}$ & $\begin{array}{l}\text { Häufigste Arzneidrogen in empfohlenen/bedingt empfohlenen } \\
\text { Hausmitteln }\end{array}$ \\
\hline Respirationssystem & 27 & 31 & Thymiankraut, Kamillenblüten, Spitzwegerichblätter, Isländisch Moos \\
\hline Reproduktion und Milchbildung (systemisch) & 20 & 82 & Brennnesselkraut, Leinsamen \\
\hline Reproduktion (lokal: intravaginal/ -uterin) & 12 & 7 & Kamillenblüten, Eichenrinde, Malvenkraut \\
\hline Urologika & 2 & 2 & Bärentraubenblätter, Preiselbeerzweige \\
\hline Haut (exkl. Klauen/Hufe, Euter/Zitzen) & 91 & 12 & $\begin{array}{l}\text { Ringelblumenblüten, Fichtenharz, Frische Johanniskrautblüten, } \\
\text { Kamillenblüten }\end{array}$ \\
\hline Klauen und Hufe & 39 & 6 & Fichtenharz, Meisterwurzwurzel, Echtes Goldrutenkraut \\
\hline Euter und Zitzen & 36 & 18 & Ringelblumenblüten, Arnikablüten, Blackenblätter \\
\hline Muskel- und Skelettsystem & 35 & 16 & Arnikablüten, Kohlblätter, Ringelblumenblüten, Wallwurzwurzel \\
\hline Antiparasitika, Insektizide und Repellentia & 15 & 28 & Lavendelkraut \\
\hline Sinnesorgane & 8 & 0 & Augentrostkraut \\
\hline Varia & 2 & 0 & Wacholderzweige (Räucherung) \\
\hline Total & 379 & 397 & \\
\hline
\end{tabular}




\section{Empirie oder Evidenz? Vor Beginn des Zeitalters der Antibiose - die tierärztliche Pharmakologie-Vorlesung 1944 von Prof. Werner Steck, Bern}

\section{Autoren: Stephan Häsler, Elisabeth Stöger}

Werner Steck (1893-1977) war während fast 40 Jahren ordentlicher Professor für spezielle Pathologie und Therapie, innere Klinik und Pharmakologie an der veterinärmedizinischen Fakultät der Universität Bern. Im Nebenamt begutachtete er für die schweizerische Arzneimittelbehörde IKS die Gesuche um Zulassung von Tierarzneimitteln. Die Pharmakologie-Vorlesung Stecks aus dem Jahr 1944 ist in einem Typoskript von 97 Seiten erhalten geblieben und wurde von cand. med. vet. Reto Gross, geboren 1923, heute Tierarzt im Ruhestand in Chur, als Nachschrift verfasst [1]. Das Lebenswerk von Steck lässt den Schluss zu, dass seine Vorlesung sowohl von Empirie als auch von Evidenz geprägt ist.

Die Vorlesung unterscheidet lokal wirkende Heilmittel (10 Gruppen) und generalisiert wirkende Heilmittel (18 Gruppen). Die Mehrheit, bezogen auf das Ausgangsmaterial, bilden die pflanzlichen Heilmittel auf der Basis von 90 Pflanzenarten. Auf der letzten Seite der Wintervorlesung führt Steck das Sulfanilamid «Prontosil» als sehr wirksam auf und erklärt, dass dieses sich an die Eiweißmoleküle der Bakterien anlagert und so das Bakterienwachstum hemmt. Die Antibiotika erwähnt er nicht. Die meisten Heilmittel müssen vom Tierarzt oder Apotheker hergestellt werden. Steck erwähnt nur wenige Markenpräparate; dabei handelt es sich vor allem um Lokalanästhetika und Antiparasitika. Therapiepläne sind nur ansatzweise vorhanden, dagegen sind Rezeptformeln und die exakten Dosierungen für die einzelnen Zieltiere angegeben. Für die Verabreichung an Pferde sind die Tagesdosen in einer Tabelle zusammengestellt, z.B. für Strychninum nitricum veratrinum $0,1 \mathrm{~g}$, Ol. therebinthinae $5 \mathrm{~g}$, Fructus juniperi $50 \mathrm{~g}$. In einer Tabelle werden die zu erwartenden Wirkungen von 28 Phytopharmaka dargestellt. Dabei muss der Leser selbst entscheiden, ob die Wirkung erwünscht ist, ob sie als Nebenwirkung in Kauf genommen wird oder ob sie Anlass zur Kontraindikation ist. Bemerkenswert ist der Hinweis auf die Geruchsbeeinträchtigung in Milch und Fleisch durch Kampher und Baldrian. Zur Reiztherapie macht er einen größeren Exkurs. Er beschreibt die Provokation der Immunreaktion durch Mikroorganismen und Stoffwechselprodukte und die unspezifische Reizsteigerung. Dazu gehören Scharfsalben, die Anwendung von Brenneisen und das Haarseil, ferner parenterale Applikationen von Gemischen auf der Basis von Jod, Terpentinöl und weiteren Stoffen.

Aus der großen Zahl von pflanzlichen Heilmitteln, die Steck aufzählt, seien 2 näher dargestellt und ihre Wirkung aus der persönlichen Erfahrung erläutert. Unter dem Begriff $\mathrm{Mu}-$ cilaginosa (= Pflanzen mit hohem Gehalt an Schleimstoffen) nennt Steck Leinsamen, Gerste, Eibischwurzel und Weitere.
Folgende Wirkungen beschreibt er: Schleimkolloide adsorbieren schädliche Stoffe infolge der großen Oberfläche, dadurch wird die Darmwand weniger geschädigt. Als Nachteil nennt er die Vergärbarkeit gewisser Substanzen und damit die Gefahr von Darmkatarrhen bei längerer Verabreichung. Laut Steck erfolgt die Anwendung zum Schutz von Haut und Schleimhaut, zur Verzögerung der Resorption, als Geschmackskorrigens und Gleitmittel sowie als Bestandteil von Pillen und Bolen. Die Indikationen decken sich mit der heute üblichen Anwendung: frisch hergestellte Leinsamenschleimzubereitung (die maximale Einweichzeit beträgt $12 \mathrm{~h}$ ) wird bei Entzündungen der Magen-Darm-Schleimhaut eingesetzt. Nach schweren Koliken und Magen-Darm-Erkrankungen wird zunächst nur die Schleimzubereitung verabreicht. Die empfohlene Tagesdosis [2] beträgt beim Pferd und Rind 50-100 g Leinsamen. Fohlen, Kälber, Schafe und Ziegen erhalten pro Tag 25-50 g, Schweine 10-25 g, Hunde 2-5 g und Katzen 1-3 g.

Die Wirkungen der Adstringentia beschreibt Steck ebenfalls sehr ausführlich: Die Gerbstoffdrogen sind zusammenziehend, bewirken eine Eiweißfällung, sind blutstillend, gefäßverengend und keimtötend. Sie werden bei Schleimhautkatarrhen und nässenden Ekzemen, als Schorfmittel und als Hämostyptika verwendet, innerlich gegen Durchfall und auch als Antidot gegen Alkaloidvergiftung. Die Eichenrinde ist nach wie vor eine viel verwendete Gerbstoffdroge. Von Steck werden auch Chinarinde, Galläpfel, Ratanhiawurzel, Tormentillwurzel und andere genannt. In der Praxis hat sich Eichenrindenabkochung bei schmierigen und nässenden Ekzemen bewährt, sowohl beim Euter-Schenkel-Ekzem der Kühe als auch bei der oberflächlichen eitrigen Hautentzündung (Hotspot) des Hundes. Einziger Nachteil ist, dass die Eichenrindenabkochung abfärbt, also Vorsicht bei hellen Hunden.

\section{Literatur}

1 Steck W: Pharmakologie. Typoskript, erstellt von Gross R. Bern, 1944. (Kopie im Archiv der Schweizerischen Vereinigung für Geschichte der Veterinärmedizin).

2 Aichberger L, Grafschafter M, Fritsch F, Gansinger D, Hagmüller W, HahnRamssl I, Hozzank A, Kolar V, Stöger E: Kräuter für Nutz- und Heimtiere. Enzersfeld, Eigenverlag, 2012.

\section{Disclosure Statement}

Die Autoren haben keine Interessenkonflikte.

Franziska Klarer: Die Studie wurde in dankenswerter Weise finanziert durch private Stiftungen (Paul Schiller Stiftung, Zürich; Bristol-Stiftung, Zürich) sowie durch die Schweizerische Medizinische Gesellschaft für Phytotherapie und den Kanton Graubünden. Die ZHAW leistete eine Anschubfinanzierung. 